\title{
BOUNDS FOR THE SOLUTIONS OF SOME DIOPHANTINE EQUATIONS IN TERMS OF DISCRIMINANTS
}

\author{
B. BRINDZA, J. H. EVERTSE and K. GYŐRY
}

(Received 19 March 1990)

Communicated by J. H. Loxton

To the memory of Professor K. Mahler

\begin{abstract}
Several effective upper bounds are known for the solutions of Thue equations, Thue-Mahler equations and superelliptic equations. One of the basic parameters occurring in these bounds is the height of the polynomial involved in the equation. In the present paper it is shown that better (and, in certain important particular cases, best possible) upper bounds can be obtained in terms of the height, if one takes into consideration also the discriminant of the polynomial.
\end{abstract}

1980 Mathematics subject classification (Amer. Math. Soc.) (1985 Revision): 11 D 41, 11 D 61.

\section{Thue equations and Thue-Mahler equations}

Let $F(X, Y)$ be a binary form of degree $n \geq 3$ with relatively prime integer coefficients and with non-zero discriminant $D(F)$. Further, let $m$ be a nonzero integer. By using his fundamental results on linear forms in logarithms, Baker [1] derived in 1968 an explicit upper bound for the absolute values of the solutions of the Thue equa tion

$$
F(x, y)=m \text { in } x, \quad y \in \mathbb{Z} \text {. }
$$

This bound depends only on $n,|m|$ and the height $H(F)$ of $F$, that is the

The first and third authors were partially supported by Grant 273 from the Hungarian National Foundation for Scientific Research. The research of the second author was made possible by a fellowship of the Royal Netherlands Academy of Arts and Science.

(C) 1991 Australian Mathematical Society 0263-6115/91 \$A2.00+0.00 
maximum of the absolute values of the coefficients of $F$. This result was extended by Coates [4] to the Thue-Mahler equation

$$
\begin{aligned}
& F(x, y)=m p_{1}^{z_{1}} \cdots p_{s}^{z_{s}} \quad \text { in } x, y, z_{1}, \ldots, z_{s} \in \mathbb{Z} \text { with } \\
&\left(x, y, p_{1} \cdots p_{s}\right)=1 \text { and } z_{1}, \ldots, z_{s} \geq 0,
\end{aligned}
$$

where $p_{1}, \ldots, p_{s}(s \geq 0)$ are distinct, fixed prime numbers. (Baker [1] and Coates [4] made the assumption that $F$ is irreducible.) The bounds obtained by Baker and Coates were later considerably improved by several authors; for references see, for example, [22], [20] and [5]. It was proved in [12] (see also [14]) that all solutions $\left(x, y, z_{1}, \ldots, z_{s}\right)$ of (2) satisfy

$$
\max \left(|x|,|y|, p_{1}^{z_{1}} \cdots p_{s}^{z_{s}}\right) \leq c_{1}(H(F) \cdot|m|)^{c_{2}}
$$

where $c_{1}, c_{2}$ are effectively computable numbers depending only on $n, s$, the maximum, say $P$, of $p_{1}, \ldots, p_{s}$ (with $P=1$ if $s=0$ ) and the splitting field, say $G$, of $F$ over $\mathbb{Q}$.

In the present paper, we show that the upper bound (3) for the solutions of (1), (2) can be replaced by another one which has a much better dependence on $H(F)$, provided that we take the discriminant $D(F)$ (which by assumption is nonzero) into consideration. Every binary form $F^{*}(X, Y)=F(a X+b Y, c X+d Y)$ with $a, b, c, d \in \mathbb{Z}$ and $a d-b c= \pm 1$, has the same discriminant as $F$. This shows that the height of a binary form can be arbitrarily large compared with its discriminant.

Let $S=\left\{p_{1}, \ldots, p_{s}\right\}$. Every $\alpha \in \mathbb{Q}$ can be expressed uniquely as $\alpha=$ $\pm p_{1}^{k_{1}} \cdots p_{2}^{k_{s}} \cdot a / b$, where $k_{1}, \ldots, k_{s}$ are rational integers and $a, b$ positive integers such that $(a, b)=1$ and $\left(a b, p_{1} \cdots p_{s}\right)=1$. We put $|\alpha|_{S}=a / b$. We call $|\alpha|_{s}$ the $S$-free part of $\alpha$. By using some effective results on $S$ unit equations (see [10], [6]) we shall prove the following theorems. Let $D_{G}$ be the discriminant of the splitting field $G$ of $F$ over $\mathbb{Q}$. Further, let $g=[G: \mathbb{Q}]$.

THEOREM 1. Let $\left(x, y, z_{1}, \ldots, z_{s}\right)$ be a solution of $(2)$. Then

$$
\max (|x|,|y|) \leq c_{3} H(F)^{3 / n}|D(F) \cdot m|_{s}^{c_{4}},
$$

where $c_{3}, c_{4}$ are effectively computable positive numbers depending only on $n, g, s, P$ and $\left|D_{G}\right|$.

The binary form $F(X, Y)$ considered above is called monic if $F(1,0)=$ 1. In the monic case, we can further improve the right-hand side of (4).

THEOREM 2. Assume that $F$ is monic. Then for each solution $\left(x, y, z_{1}\right.$, $\ldots, z_{s}$ ) of (2) we have

$$
|y| \leq c_{S}|D(F) \cdot m|_{S}^{c_{6}}
$$


and

$$
|x| \leq c_{7}\left(|D(F)|^{1 / n(n-1)}+|F(0,1)|^{1 / n}\right) \cdot|D(F) \cdot m|_{S}^{c_{8}},
$$

where $c_{5}, c_{6}, c_{7}, c_{8}$ are effectively computable numbers depending only on $n, g, s, P$ and $\left|D_{G}\right|$.

Observe that the bound in (5) is independent of $H(F)$. The following example shows that, in (6), the dependence of $|D(F)|$ cannot be dropped. Let in particular $S=\{2\}$, let $\left(x_{0}, y_{0}\right) \in \mathbb{Z}^{2}$ with $x_{0} y_{0} \neq 0,\left(x_{0}, y_{0}, 2\right)=1$ and $F\left(x_{0}, y_{0}\right)=m$, and let $F_{k}^{*}(X, Y)=F\left(X, 2^{k} Y\right)$ for $k=1,2, \ldots$ Then $\left|D\left(F_{k}^{*}\right)\right|_{S}=|D(F)|_{S}$. Further, $x=2^{k} x_{0}, y=y_{0}, z=n k$ is a solution of $F_{k}^{*}(x, y)=m \cdot 2^{z}$ in $x, y, z \in \mathbb{Z}$ with $(x, y, z)=1$ and $z \geq 0$. This shows that for large $k,|x|$ cannot be estimated from above by a bound which depends on $\left|D\left(F_{k}^{*}\right)\right|_{S}$ but not on $\left|D\left(F_{k}^{*}\right)\right|$.

We now consider equation (1);S is still a set of prime numbers, the largest of which is $P$.

THEOREM 3. Assume that $F$ is monic. Then every solution $(x, y)$ of (1) satisfies

$$
|y| \leq c_{9}|m|^{1 / n}|D(F) \cdot m|_{S}^{c_{10}}
$$

and

$$
|x| \leq c_{11}|m|^{1 / n}\left(1+|F(0,1)|^{1 / n}\right)|D(F) \cdot m|_{S}^{c_{12}},
$$

where $c_{9}, c_{10}, c_{11}, c_{12}$ are effectively computable numbers depending only on $n, g, s, P$ and $\left|D_{G}\right|$.

We can compare (7) with a recent estimate of Brindza [3]; he derived the sharper estimate

$$
|y| \leq c(n) H(F)^{(2 n-3)(n-2)}
$$

for the solutions $(x, y) \in \mathbb{Z}^{2}$ of $(1)$; however, he made the assumptions that $m=1$, that $F$ is monic and irreducible and that the unit rank of $G$ is less than $n-1$.

The following example shows that the exponent $1 / n$ of $|F(0,1)|$ in (6) and (8) are already best possible. Let $m=a^{n}$ for some $a \in \mathbb{N}$, let $P(X, Y)=X^{n}+Y^{n}$ and let $F_{k}(X, Y)=P(X+k Y, Y)$ for $k=1,2, \ldots$ Then $D\left(F_{k}\right)=D(P), F_{k}$ and $P$ have the same splitting field over $\mathbb{Q}$, $x=-k a, y=a$ is a solution of $F_{k}(x, y)=m$ and, for large $k, H\left(F_{k}\right)=$ $\left|F_{k}(0,1)\right|=k^{n}+1$ and $|x| \geq|m / 2|^{1 / n}\left|F_{k}(0,1)\right|^{1 / n}$.

It is possible to reduce the number of parameters on which the bounds in Theorems 1, 2 and 3 depend. Obviously, $g \leq n !$. Further, from prime 
number theory it follows that $s \leq 2 P / \log P$ (cf. [17]). Finally, in Section 3 we shall prove that

$$
\left|D_{G}\right| \leq(n P)^{s n g}|D(F)|_{S}^{g} .
$$

When we combine these inequalities for instance with Theorem 1, we get that every solution $\left(x, y, z_{1}, \ldots, z_{s}\right)$ of (2) satisfies

$$
\max (|x|,|y|) \leq c_{13} H(F)^{3 / n} \cdot|m|_{S}^{c_{14}},
$$

where $c_{13}, c_{14}$ are effectively computable numbers depending only on $n, P$ and $|D(F)|_{S}$. We remark that the upper bound in $(10)$ is no longer polynomial in $|D(F)|_{S}$. Similar consequences can be deduced from Theorems 2 and 3.

By a result of Györy [8] we have

$$
n=\operatorname{deg}(F) \leq 3+\frac{2}{\log 3} \log |D(F)| .
$$

By inserting this bound into (4) with $S=\varnothing$ and using again $g \leq n !$ and (9), we get that every solution $(x, y)$ of (1) satisfies

$$
\max (|x|,|y|) \leq c_{15} H(F)^{3 / n}|m|^{c_{16}},
$$

where $c_{15}, c_{16}$ are effectively computable and depend only on $|D(F)|$. Again, it is possible to derive a similar consequence from Theorem 3 .

In Theorems 1, 2 and 3, it is possible to replace $|D(F)|_{S}$ by some number depending only on the largest prime factor $P(D(F))$ of $D(F)$. Let $S^{\prime}$ be the set consisting of $p_{1}, \ldots, p_{s}$ and the primes dividing $D(F)$. By applying (10) with $S^{1}$ instead of $S$ we get

Corollary 1. Every solution $\left(x, y, z_{1}, \ldots, z_{s}\right)$ of (2) satisfies

$$
\max (|x|,|y|) \leq c_{17} H(F)^{3 / n}|m|_{S^{\prime}}^{c_{18}},
$$

where $c_{17}, c_{18}$ are effectively computable numbers depending only on $n, P$ and $P(D(F))$.

Again, Theorems 2 and 3 have similar consequences.

We note that the above-mentioned results of [10] and [6] which provide the main tools in the proofs of Theorems 1,2 and 3 above were all proved by means of the theory of linear forms in logarithms and its $p$-adic analogue. Further, these results in [10] and [6] were established in a more general situation, over algebraic number fields. By using these more general versions 
from [10] and [6], the results of this section can be easily extended to the algebraic number field case.

\section{Super-elliptic equations}

Let $f(x) \in \mathbb{Z}[X]$ be a polynomial of degree $n$ with at least two distinct roots, and consider the super-elliptic equation

$$
f(x)=y^{z} \quad \text { in } x, y, z \in \mathbb{Z} \quad \text { with }|y|>1, z>1 .
$$

By using the theory of linear forms in logarithms, Schinzel and Tijdeman [18] proved that for all solutions of (13), $z<c_{19}$ where $c_{19}$ is an effectively computable number depending only on $n$ and the height $H(f)$ of $f$. For various generalizations and related results, we refer to [20] and [24]. In the case when $f$ is monic and its discriminant $D(f)$ is different from zero, it follows from a theorem of Györy [9] (see also Lemma 3 in Section 4) that $f(X)=f^{*}(X+a)$ with some $a \in \mathbb{Z}$ and $f^{*} \in \mathbb{Z}[X]$ for which $H\left(f^{*}\right) \leq c_{20}$ where $c_{20}$ is an effectively computable number depending only on $n$ and $|D(f)|$. Then (13) implies $f^{*}(x+a)=y^{z}$ for each solution and, by the theorem of Schinzel and Tijdeman, $z$ is less than some effectively computable number which depends only on $n$ and $|D(f)|$, and not on $H(f)$. This gives a much better estimate for $z$ if $H(f)$ is large with respect to $|D(f)|$.

In our paper, we shall deal with equation (13) in the important special case when $f$ is irreducible. It is straightforward, but technically more complicated, to generalize our results to arbitrary polynomials $f \in \mathbb{Z}[X]$ with no multiple zeros. By applying a recent estimate of Philippon and Waldschmidt [16] for linear forms in logarithms, we shall derive upper bounds for $z$ which depend not only on $n$ and $H(f)$ but also on $|D(f)|$. For latter applications, the upper bounds will be given in completely explicit form.

Theorem 4. Suppose that $f(X) \in \mathbb{Z}[X]$ is an irreducible monic polynomial of degree $n \geq 2$. Then all solutions of (13) satisfy

$$
z<\max \left\{n \log _{2}(2 H(F)+3), c_{21}|D(f)|^{2}\right\}
$$

with some $c_{21} \geq 2^{20(n+3)}(n+2)^{15 n}$.

Theorem 4 and Lemma 3 imply immediately

Theorem 5. Suppose that $f \in \mathbb{Z}[X]$ is an irreducible monic polynomial of degree $n \geq 2$. Then all solutions of (13) satisfy

$$
z<\left(6 n^{3}\right)^{30 n^{3}} \cdot|D(f)|^{5 n^{2}}
$$


The proof depends on an effective estimate in Lemma 3 which can be improved in terms of $n$ by using some recent effective estimates for linear forms in logarithms.

It follows from a result of Györy [8, Theorem 1 and Remark 1.2] that if $f \in \mathbb{Z}[X]$ is monic and irreducible with discriminant $|D(f)| \leq D\left(D \geq e^{e}\right)$ then

$$
\operatorname{deg}(f) \leq \log D .
$$

Hence, estimating $z$, we can eliminate the dependence on $n$ and we obtain from Theorem 5 the following

Corollary 2. Suppose that $f \in \mathbb{Z}[X]$ is an irreducible monic polynomial of degree at least 2 with $|D(f)| \leq D\left(D \geq e^{e}\right)$. Then all solutions of (13) satisfy

$$
\log z<100(\log D)^{3} \log \log D \text {. }
$$

It is easy to see that if $x, y, z$ is a solution of (13) then, for $|x|$, there exists no upper bound depending only on $|D(f)|$. However, combining Corollary 2 with Lemma 3, (14) and a result of Baker [2] on super-elliptic equations, we have

THEOREM 6. If $f \in \mathbb{Z}[X]$ is an irreducible monic polynomial of degree at least 2 , then all solutions of (13) satisfy $|y|^{z}<c_{22}$, where $c_{22}$ is an effectively computable number depending only on $|D(f)|$.

In fact, Baker's theorem applies to (13) only if $n=\operatorname{deg}(f) \geq 3$ or $n=$ $2, z \geq 3$. However, it is easy to see that if $n=z=2$ then $|y|^{2} \leq|D(f)|^{2}$ and hence our Theorem 6 is true in the present form.

In other words, Theorem 6 says that the power values of an irreducible monic polynomial $f \in \mathbb{Z}[X]$ of degree at least two are bounded by an effectively computable number which depends only on the discriminant of $f$.

\section{Proofs of Theorems 1, 2 and 3}

In this section, we shall prove Theorems 1,2 and 3 and the inequality (9). To do so, we shall need some further notation and one lemma.

Let $G$ be an algebraic number field of degree $g$ with ring of integers $O_{G}$. Let $M_{G}$ denote the set of places (equivalence classes of multiplicative valuations) on $G$. In every place $v$ we choose a valuation $|\cdot|_{v}$ in the following way: if $v$ is an infinite place corresponding to an embedding $\sigma: G \rightarrow \mathbb{C}$ then 
we put, for every $\alpha \in G,|\alpha|_{v}=|\sigma(\alpha)|^{1 / g}$ or $|\alpha|_{v}=|\sigma(\alpha)|^{2 / g}$, according as $\sigma(G) \subset \mathbb{R}$ or $\sigma(G) \not \subset \mathbb{R}$; if $v$ is a finite place corresponding to the prime ideal $\mathfrak{p}$ in $G$ then we put $|\alpha|_{v}=N_{G / \mathbb{Q}}(\mathfrak{p})^{-\operatorname{ord}_{\mathfrak{p}}(\alpha) / g}$ for $\alpha \in G \backslash\{0\}$ and $|0|_{v}=0$. The valuations thus defined satisfy the product formula

$$
\prod_{v \in M_{G}}|\alpha|_{v}=1 \text { for } \alpha \in G \backslash\{0\} .
$$

Further, for a vector $\mathrm{a}=\left(a_{1}, \ldots, a_{N}\right) \in G^{N}$ and for $v \in M_{G}$, we put

$$
|\mathbf{a}|_{v}=\max \left(\left|a_{1}\right|_{v}, \ldots,\left|a_{N}\right|_{v}\right)
$$

and we define the homogeneous height of $\mathbf{a}$ by

$$
h^{*}(\mathbf{a})=\prod_{v \in M_{G}}|\mathbf{a}|_{v}
$$

We note that, by (15), $h^{*}(\lambda \mathbf{a})=h^{*}(\mathbf{a})$ for every $\lambda \in G \backslash\{0\}$. For $\alpha \in G$, we define the absolute height $h(\alpha)$ of $\alpha$ by $h(\alpha)=h^{*}(1, \alpha)$. The absolute height has the following elementary properties:

$$
h\left(\alpha^{-1}\right)=h(\alpha) \quad \text { for } \alpha \in G \backslash\{0\}
$$

and

$$
h(\alpha \cdot \beta) \leq h(\alpha) h(\beta), \quad h(\alpha+\beta) \leq 2 h(\alpha) h(\beta) \quad \text { for } \alpha, \beta \in G .
$$

If $Q \in G\left[X_{1}, \ldots, X_{N}\right]$ is any polynomial then the homogeneous height $h^{*}(Q)$ of $Q$ is defined as the homogeneous height of the vector consisting of the coefficients of $Q$. Further, we define $|Q|_{v}$ as the maximum of the $v$-values of the coefficients of $Q$. We note that $h^{*}(a), h(\alpha)$ and $h^{*}(Q)$ depend only on a, $\alpha$ and $Q$, and are independent of the choice of the number field $G$. Finally, for any nonzero algebraic number $\alpha$ with minimal polynomial $a_{0} \prod_{i=1}^{n}\left(X-\alpha_{i}\right) \in \mathbb{Z}[X]$, we have, by [15, page 54$]$, that

$$
h(\alpha)=\left(\left|a_{0}\right| \cdot \prod_{i=1}^{n} \max \left(1,\left|\alpha_{i}\right|\right)\right)^{1 / n}
$$

and (see, for example, [7]) that

$$
2^{1-n} H(\alpha) \leq(h(\alpha))^{n} \leq \sqrt{n+1} \cdot H(\alpha),
$$

where $H(\alpha)$ denotes the usual height of $\alpha$, that is, the maximum absolute value of the coefficients of the minimal defining polynomial of $\alpha$ over $\mathbb{Z}$. If in particular $\alpha \in \mathbb{Q}$, then $h(\alpha)$ coincides with $H(\alpha)$.

Let $T$ be a finite set of places on $G$ containing $t$ finite places. Denote by $O_{T}$ the ring of $T$-integers:

$$
O_{T}=\left\{\alpha \in G:|\alpha|_{v} \leq 1 \text { for } v \in M_{G} \backslash T\right\} .
$$


By an $O_{T}$-ideal we mean a finitely generated $O_{T}$-module contained in $G$; an $O_{T}$-ideal contained in $O_{T}$ is called integral. Let $\mathfrak{p}_{1}, \ldots, \mathfrak{p}_{t}$ be prime ideals corresponding to the finite places in $T$, and let $P$ be the maximum of the rational primes lying below $\mathfrak{p}_{1}, \ldots, \mathfrak{p}_{t}$ (with $P=1$ if $t=0$ ). Then, for every $O_{T}$-ideal $\mathfrak{a}$ there is a unique $O_{G}$-ideal $\mathfrak{a}^{*}$, composed of prime ideals outside $\left\{\mathfrak{p}_{1}, \ldots, \mathfrak{p}_{t}\right\}$, such that $\mathfrak{a}=\mathfrak{a}^{*} O_{T}$. We put

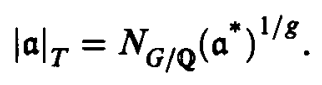

If $\mathfrak{a}$ is any $O_{T}$-ideal and $\mathfrak{b}, \mathfrak{c}$ are integral $O_{T}$-ideals such that $\mathfrak{a}=\mathfrak{b c}^{-1}$, $\mathfrak{b}+\mathfrak{c}=O_{T}$, then we put

$$
n_{T}(\mathfrak{a})=|\mathfrak{b}|_{T} \cdot|\mathfrak{c}|_{T}
$$

In particular, if $\mathfrak{a}$ is the $O_{T}$-ideal generated by $\alpha$ then we put

$$
|\alpha|_{T}=|\mathfrak{a}|_{T}, \quad n_{T}(\alpha)=n_{T}(\mathfrak{a}) .
$$

It is not difficult to prove that

$$
|\alpha|_{T}=\prod_{v \in T}|\alpha|_{v}, \quad n_{T}(\alpha)=\prod_{v \in M_{G} \backslash T} \max \left(|\alpha|_{v},|\alpha|_{v}^{-1}\right)
$$

We shall use frequently the fact that if $h_{G}, R_{G}$ and $D_{G}$ denote the class number, regulator and discriminant of $G$ then

$$
h_{G} R_{G} \leq 4 e^{g-1}\left|D_{G}\right|^{1 / 2}\left(\log \left|D_{G}\right|\right)^{g-1} \text { and } R_{G} \geq 0.056 .
$$

The first inequality is due to Siegel [21], the second one to Zimmert [25].

The proof of Theorem 1 is based on the following lemma, in which $c_{23}, c_{24}$ and $c_{25}$ denote effectively computable positive numbers depending only on $t, P, g$ and $\left|D_{G}\right|$.

LEMMA 1. Let $\mathbf{u}=(u, v) \in(G \backslash\{0\})^{2}$ with $u+v=1$ and $n_{T}(u) \leq A$, $n_{T}(v) \leq A$. Then we have $h^{*}(\mathbf{u}) \leq c_{23} A^{c_{24}}$.

Proof. There is an integral $O_{T}$-ideal $\mathfrak{a}$ such that $u \mathfrak{a}$ and $v \mathfrak{a}$ are integral, and that $|\mathfrak{a}|_{T} \leq A^{2}$. By [6, Lemma 3]., it is possible to choose $\alpha \in \mathfrak{a}$ with $\alpha \neq 0$ such that $|\alpha|_{T} \leq c_{25}|a|_{T} \leq c_{25} A^{2}$. Putting $u^{\prime}=\alpha u, v^{\prime}=\alpha v, w^{\prime}=-\alpha$ we have $u^{\prime}+v^{\prime}+w^{\prime}=0, u^{\prime}, v^{\prime}, w^{\prime} \in O_{T}$ and $\max \left(\left|u^{\prime}\right|_{T},\left|v^{\prime}\right|_{T},\left|w^{\prime}\right|_{T}\right) \leq$ $c_{25} A^{3}$. The lemma now follows from [6, Lemma 11] which was a direct consequence of [10, Lemma 6].

In what follows, $c_{26}, c_{27}, \ldots, c_{42}$ are numbers of the form $c^{\prime}|D(F) m|_{S}^{c^{\prime \prime}}$, where $c^{\prime}, c^{\prime \prime}$ are effectively computatble positive numbers depending only on $n, s, P, g$ and $\left|D_{G}\right|$. Consider the binary form $F(\mathbf{X})=F(X, Y)$ of degree $n$ occurring in Theorem 1 , and suppose that $G$ is the splitting field 
of $F$ over $\mathbb{Q}$ with the parameters $g, D_{G}$ specified above. Let $T$ denote now the finite set of places on $G$ lying above those in $S$ and the absolute value on $\mathbb{Q}$, and let $t$ be the number of finite places in $T$. Then $t \leq g \cdot s$. Further, for every $\alpha \in \mathbb{Q}$ we have $|\alpha|_{T}=|\alpha|_{S}$.

PROOF OF THEOREM 1. It suffices to prove Theorem 1 for solutions $(x, y$, $\left.z_{1}, \ldots, z_{s}\right)$ with $(x, y)=1$. Indeed, assume that Theorem 1 holds for solutions with $(x, y)=1$. If $\left(x, y, z_{1}, \ldots, z_{s}\right)$ is an arbitrary solution with $\left(x, y, p_{1}, \ldots, p_{s}\right)=1$ and with $(x, y)=d$, say, then

$$
\max (|x / d|,|y / d|) \leq c_{3} H(F)^{3 / n}\left|D(F) m / d^{n}\right|_{S}^{c_{4}},
$$

which implies that $\max (|x|,|y|) \leq c_{3} H(F)^{3 / n}|D(F) m|_{S}^{c_{4}}$, provided that $c_{4} \geq$ $1 / n$.

We can express $F(\mathbf{X})$ as

$$
F(\mathbf{X})=\prod_{i=1}^{n} l_{i}(\mathbf{X})
$$

where each $l_{i}(\mathbf{X})$ is a linear form in $X, Y$ with coefficients in $G$. Let $\Delta_{i j}=$ $\operatorname{det}\left(l_{i}, l_{j}\right)$ be the coefficient determinant of $l_{i}$ and $l_{j}$. Since, by assumption, $D(F) \neq 0$, we have $\Delta_{i j} \neq 0$ for all distinct $i, j \in\{1, \ldots, n\}$. Further, we have, for all distinct $i, j, k \in\{1, \ldots, n\}$, that

$$
\Delta_{j k} l_{i}(\mathbf{X})+\Delta_{k i} l_{j}(\mathbf{X})+\Delta_{i j} l_{k}(\mathbf{X})=0
$$

identically in $\mathbf{X}$.

Let now $\mathbf{x}=(x, y)$ be an arbitrary but fixed pair of integers such that $(x, y)=1$ and $\left(x, y, z_{1}, \ldots, z_{s}\right)$ is a solution of (2) for some non-negative integers $z_{1}, \ldots, z_{s}$. Put

$$
u=\frac{-\Delta_{j k} l_{i}(\mathbf{x})}{\Delta_{i j} l_{k}(\mathbf{x})}, \quad v=\frac{-\Delta_{k i} l_{j}(\mathbf{x})}{\Delta_{i j} l_{k}(\mathbf{x})} .
$$

Then we get

$$
u+v=1 .
$$

We claim that

$$
n_{T}(u) \leq|D(F) \cdot m|_{S}, \quad n_{T}(v) \leq|D(F) \cdot m|_{S} .
$$

Let $\left(l_{i}\right)_{T}$ be the $O_{T}$-ideal generated by the coefficients of $l_{i}$ for $i=1, \ldots, n$, and, for $\alpha \in G$, let $(\alpha)_{T}$ be the $O_{T}$-ideal generated by $\alpha$. Put

$$
\mathfrak{d}_{i j}=\left(\Delta_{i j}\right)_{T} /\left(l_{i}\right)_{T}\left(l_{j}\right)_{T}, \quad a_{i}(\mathbf{x})=\left(l_{i}(\mathbf{x})\right)_{T} /\left(l_{i}\right)_{T}
$$


for all distinct $i, j \in\{1, \ldots, n\}$. Then the $O_{T}$-ideals $\mathfrak{d}_{i j}$ and $\mathfrak{a}_{i}(\mathbf{x})$ are integral. Further, we have $\prod_{i \neq j} \mathfrak{d}_{i j} \mid(D(F))_{T}$ and $\prod_{i=1}^{n} \mathfrak{a}_{i}(\mathbf{x}) \mid(F(\mathbf{x}))_{T}$ in $O_{T}$ and

Hence

$$
(u)_{T}=\frac{\mathfrak{d}_{j k} \mathfrak{a}_{i}(\mathbf{x})}{\mathfrak{d}_{i j} \mathfrak{a}_{k}(\mathbf{x})}
$$

$$
\begin{aligned}
n_{T}(u) & \leq\left|\mathfrak{d}_{j k}\right|_{T} \cdot\left|\mathfrak{a}_{i}(\mathbf{x})\right|_{T} \cdot\left|\mathfrak{d}_{i j}\right|_{T} \cdot\left|\mathfrak{a}_{k}(\mathbf{x})\right|_{T} \\
& \leq|D(F)|_{T} \cdot|F(\mathbf{x})|_{T}=|D(F)|_{S} \cdot|F(\mathbf{x})|_{S} \leq|D(F) \cdot m|_{S} .
\end{aligned}
$$

The inequality for $n_{T}(v)$ follows in the same way.

By Lemma 1 and the product formula we have

$$
h^{*}\left(\Delta_{j k} l_{i}(\mathbf{x}), \Delta_{k i} l_{j}(\mathbf{x})\right)=h^{*}(u, v) \leq c_{26} \text {. }
$$

Put

$$
\begin{gathered}
u^{\prime}=\Delta_{j k} l_{i}(\mathbf{x}), \quad v^{\prime}=\Delta_{k i} l_{j}(\mathbf{x}), \\
l_{i}(\mathbf{x})=\alpha_{i} x+\beta_{i} y, \quad \text { and } l_{j}(\mathbf{x})=\alpha_{j} x+\beta_{j} y .
\end{gathered}
$$

Then if

we have, by Cramer's rule,

$$
\kappa=\operatorname{det}\left(\begin{array}{cc}
\Delta_{j k} \alpha_{i} & \Delta_{j k} \beta_{i} \\
\Delta_{k i} \alpha_{j} & \Delta_{k i} \beta_{j}
\end{array}\right),
$$

$$
\kappa x=\Delta_{k i} \beta_{j} u^{\prime}-\Delta_{j k} \beta_{i} v^{\prime}, \quad \kappa y=-\Delta_{k i} \alpha_{j} u^{\prime}+\Delta_{j k} \alpha_{i} v^{\prime} .
$$

Let $\omega(v)=\log \max \left(1,|2|_{v}\right) / \log 2$ for $v \in M_{G}$. Then

$$
\begin{aligned}
|\kappa x|_{v} & \leq 2^{\omega(v)} \max \left(\left|\Delta_{k i} \beta_{j}\right|_{v},\left|\Delta_{j k}, \beta_{i}\right|_{v}\right) \cdot \max \left(\left|u^{\prime}\right|_{v},\left|v^{\prime}\right|_{v}\right) \\
& \leq 4^{\omega(v)}\left|l_{i}\right|_{v}\left|l_{j}\right|_{v}\left|l_{k}\right|_{v} \max \left(\left|u^{\prime}\right|_{v},\left|v^{\prime}\right|_{v}\right) .
\end{aligned}
$$

We can derive the same upper bound for $|\kappa y|_{v}$. By taking the product over all $v \in M_{G}$ and using (21) and the product formula, we get

$$
\begin{gathered}
h^{*}(\mathbf{x})=h^{*}(\kappa x, \kappa y) \leq\left(\prod_{v \in M_{G}} r^{\omega(v)}\left|l_{i}\right|_{v}\left|l_{j}\right|_{v}\left|l_{k}\right|_{v}\right) \\
\cdot h^{*}\left(u^{\prime}, v^{\prime}\right) \leq 4 h^{*}\left(l_{i}\right) h^{*}\left(l_{j}\right) h^{*}\left(l_{k}\right) c_{26} .
\end{gathered}
$$

Further, we have [15, Ch. III, Proposition 2.4] that

$$
\prod_{i=1}^{n} h^{*}\left(l_{i}\right) \leq c_{27} h^{*}(F)
$$

Hence we can choose $i, j, k$ such that

$$
h^{*}\left(l_{i}\right) h^{*}\left(l_{j}\right) h^{*}\left(l_{k}\right) \leq c_{28} h^{*}(F)^{3 / n} \text {. }
$$


By inserting this into (22), we obtain

$$
h^{*}(\mathbf{x}) \leq c_{29} h^{*}(F)^{3 / n} .
$$

Since $(x, y)=1$, we have $h^{*}(\mathbf{x})=\max (|x|,|y|)$. Further, by assumption, $F$ has relatively prime rational integer coefficients, and hence $h^{*}(F)=$ $H(F)$. Now Theorem 1 follows at once from (24).

Proof of Theorems 2 AND 3. We shall prove Theorems 2 and 3 simultaneously. By a similar argument to that in the beginning of the proof of Theorem 1 , it follows that we can restrict ourselves to solutions with $(x, y)=1$. So let $\left(x, y, z_{1}, \ldots, z_{s}\right)$ (with $z_{1}=\cdots=z_{s}=0$ when we are proving Theorem 3 ) be an arbitrary but fixed solution of $(2)$ with $(x, y)=1$. Then we have

$$
F(x, y)=m p_{1}^{z_{1}} \cdots p_{s}^{z_{s}}= \pm|m|_{S} p_{1}^{w_{1}} \cdots p_{s}^{w_{s}} z^{n}
$$

where $w_{i}$ is an integer with $0 \leq w_{i} \leq n-1$ for $i=1, \ldots, s$ and $z$ is an integer composed of primes from $S=\left\{r_{1}, \ldots, p_{s}\right\}$. Put $m^{\prime}= \pm|m|_{S} p_{1}^{w_{1}} \cdots p_{s}^{w_{s}}$, $x^{\prime}=x / z$ and $y^{\prime}=y / z$. Then (25) becomes

$$
F\left(x^{\prime}, y^{\prime}\right)=m^{\prime} \text {. }
$$

Note that

$$
|m|_{S}=\left|m^{\prime}\right|_{S} \leq\left|m^{\prime}\right| \leq P^{n s}|m|_{S}
$$

Since $F$ is monic, we have $F(X, Y)=\prod_{i=1}^{n}\left(X+\beta_{i} Y\right)$, whence

$$
\prod_{i=1}^{n}\left(x^{\prime}+\beta_{i} y^{\prime}\right)=m^{\prime}
$$

for certain distinct $\beta_{1}, \ldots, \beta_{n} \in O_{G}$. For $i, j, k \in\{1, \ldots, n\}$ with $i \neq$ $j \neq k \neq i$, put

$$
u_{i j k}=\frac{\beta_{j}-\beta_{k}}{\beta_{j}-\beta_{i}} \cdot \frac{x^{\prime}+\beta_{i} y^{\prime}}{x^{\prime}+\beta_{k} y^{\prime}}, \quad v_{i j k}=\frac{\beta_{k}-\beta_{i}}{\beta_{j}-\beta_{i}} \cdot \frac{x^{\prime}+\beta_{j} y^{\prime}}{x^{\prime}+\beta_{k} y^{\prime}} .
$$

Then we have Siegel's identity

$$
u_{i j k}+v_{i j k}=1 \text {. }
$$

Note that $\beta_{p}-\beta_{q} \mid D(F)$ in $O_{T}$ for distinct $p, q \in\{1, \ldots, n\}$. Further, by (27), we have $x^{\prime}+\beta_{p} y^{\prime} \mid m^{\prime}$ in $O_{T}$ for $p \in\{1, \ldots, n\}$. Together with (26), this implies that

$$
n_{T}\left(u_{i j k}\right) \leq\left|\beta_{j}-\beta_{k}\right|_{T}\left|x^{\prime}+\beta_{i} y^{\prime}\right|_{T}\left|\beta_{j}-\beta_{i}\right|_{T}\left|x^{\prime}+\beta_{k} y^{\prime}\right|_{T} \leq c_{30}
$$

One can derive a similar upper bound for $n_{T}\left(v_{i j k}\right)$. Therefore, by Lemma 1 ,

$$
h^{*}\left(u_{i j k}, v_{i j k}\right) \leq c_{31}
$$


which is the same as

(29) $h\left(\frac{\left(\beta_{j}-\beta_{k}\right)\left(x^{\prime}+\beta_{i} y^{\prime}\right)}{\left(\beta_{k}-\beta_{i}\right)\left(x^{\prime}+\beta_{j} y^{\prime}\right)}\right) \leq c_{31}$ for $i, j, k \in\{1, \ldots, n\}$ with $k \neq i$.

From the identity

$$
\frac{\beta_{j}-\beta_{k}}{\beta_{j}-\beta_{i}}+\frac{\beta_{k}-\beta_{i}}{\beta_{j}-\beta_{i}}=1
$$

from $\beta_{p}-\beta_{q} \mid D(F)$ for $p, q \in\{1, \ldots, n\}$ and from Lemma 1 , it follows that

$$
h\left(\frac{\beta_{j}-\beta_{k}}{\beta_{k}-\beta_{i}}\right) \leq c_{32} \quad \text { for } i, j, k \in\{1, \ldots, n\} \text { with } k \neq i .
$$

By combining this with (29), we get

$$
h\left(\frac{x^{\prime}+\beta_{i} y^{\prime}}{x^{\prime}+\beta_{j} y^{\prime}}\right) \leq c_{33} \text { for } i, j \in\{1, \ldots, n\} .
$$

Using (27), we get

$$
h\left(\frac{\left(x^{\prime}+\beta_{i} y^{\prime}\right)^{n}}{m^{\prime}}\right) \leq h\left(\prod_{j=1}^{n} \frac{x^{\prime}+\beta_{i} y^{\prime}}{x^{\prime}+\beta_{j} y^{\prime}}\right) \leq \prod_{j=1}^{n} h\left(\frac{x^{\prime}+\beta_{i} y^{\prime}}{x^{\prime}+\beta_{j} y^{\prime}}\right) \leq c_{34} .
$$

Together with (26) this implies that

$$
h\left(x^{\prime}+\beta_{i} y^{\prime}\right) \leq h\left(m^{\prime}\right)^{1 / n} c_{35}=\left|m^{\prime}\right|^{1 / n} c_{35} \leq c_{36} .
$$

By applying (32) for two different subscripts $i, j$ we get

$$
h\left(\left(\beta_{i}-\beta_{j}\right) y^{\prime}\right) \leq 2 h\left(x^{\prime}+\beta_{i} y^{\prime}\right) h\left(x^{\prime}+\beta_{j} y^{\prime}\right) \leq c_{37} .
$$

We remark that $(y, z)=1$ (in $\mathbb{Z}$ ), since $z \mid F(x, y), F(x, y)=x^{n}+y(\quad$ ) and $(x, y)=1$. In general, if $\alpha \in G \backslash\{0\}$ and $[\alpha]=\mathfrak{a b}^{-1}$ (here [ ] is used to denote $O_{T}$-ideals), where $\mathfrak{a}, \mathfrak{b}$ are integral $O_{G}$-ideals such that $\mathfrak{a}+\mathfrak{b}=[1]$, then

$$
h(\alpha) \geq \max \left(N_{G / \mathbb{Q}}(\mathfrak{a}), N_{G / \mathbf{Q}}(\mathfrak{b})\right)^{1 / g} \quad(g=[G: \mathbb{Q}]) .
$$

For $\alpha=\left(\beta_{i}-\beta_{j}\right) y^{\prime}$ we can take $\mathfrak{a}=\left(\left[\beta_{i}-\beta_{j}\right] /\left[\beta_{i}-\beta_{j}, z\right]\right)[y]$ and $\mathfrak{b}=$ $[z] /\left[\beta_{i}-\beta_{j}, z\right]$, since $y^{\prime}=y / z,(y, z)=1$ and $\beta_{i}-\beta_{j} \in O_{G}$. This implies that

$$
h\left(\left(\beta_{i}-\beta_{j}\right) y^{\prime}\right) \geq N_{G / \mathbb{Q}}(\mathfrak{a})^{1 / g}=N_{G / \mathbb{Q}}\left(\frac{\left[\beta_{i}-\beta_{j}\right]}{\left[\beta_{i}-\beta_{j}, z\right]}\right)^{1 / g} \cdot|y| \geq|y|
$$

and

$$
\begin{aligned}
h\left(\left(\beta_{i}-\beta_{j}\right) y^{\prime}\right) & \geq N_{G / \mathbf{Q}}(\mathfrak{b})^{1 / g}=N_{G / \mathbf{Q}}\left(\frac{[z]}{\left[\beta_{i}-\beta_{j}, z\right]}\right)^{1 / g} \\
& \geq \frac{|z|}{\left|N_{G / \mathbf{Q}}\left(\beta_{i}-\beta_{j}\right)\right|^{1 / g}} \geq \frac{|z|}{h\left(\beta_{i}-\beta_{j}\right)} .
\end{aligned}
$$


By combining (33) and (34) we get

$$
|y| \leq c_{37}
$$

This proves (5) (of Theorem 2) and (7) (of Theorem 3; note that the term $|m|^{1 / n}$ disappears when $\left.(x, y)=1\right)$. In order to estimate $|z|$, we have to determine an upper bound for $h\left(\beta_{i}-\beta_{j}\right)$. By (30) we have for all $i, j, k, l \in$ $\{1, \ldots, n\}(i \neq j, l \neq k)$, by interchanging some of the subscripts, that

$$
\begin{aligned}
h\left(\frac{\beta_{i}-\beta_{j}}{\beta_{l}-\beta_{k}}\right) & =h\left(\frac{\beta_{i}-\beta_{l}}{\beta_{l}-\beta_{k}}-\frac{\beta_{j}-\beta_{l}}{\beta_{l}-\beta_{k}}\right) \\
& \leq 2 h\left(\frac{\beta_{i}-\beta_{l}}{\beta_{l}-\beta_{k}}\right) h\left(\frac{\beta_{j}-\beta_{l}}{\beta_{l}-\beta_{k}}\right) \leq c_{38} .
\end{aligned}
$$

Hence

$$
\begin{aligned}
h\left(\frac{\left(\beta_{i}-\beta_{j}\right)^{n(n-1)}}{D(F)}\right) & =h\left(\prod_{l \neq k} \frac{\beta_{i}-\beta_{j}}{\beta_{l}-\beta_{k}}\right) \\
& \leq \prod_{l \neq k} h\left(\frac{\beta_{i}-\beta_{j}}{\beta_{l}-\beta_{k}}\right) \leq c_{39} .
\end{aligned}
$$

This implies that

$$
h\left(\beta_{i}-\beta_{j}\right) \leq h(D(F))^{1 / n(n-1)} c_{40}=|D(F)|^{1 / n(n-1)} c_{40} .
$$

By combining this with (33) and (35) we get

$$
|z| \leq h\left(\beta_{i}-\beta_{j}\right) h\left(\left(\beta_{i}-\beta_{j}\right) y^{\prime}\right) \leq|D(F)|^{1 / n(n-1)} c_{41} .
$$

We now estimate $|x|$ from above. Fix an extension $|$.$| to G$ of the ordinary absolute value on $\mathbb{Q}$. Choose $\beta_{i}$ such that either $\beta_{i}=0$ (if $F(0,1)=0$ ) or $\left|\beta_{i}\right| \leq|F(0,1)|^{1 / n}$ (if $\left.F(0,1) \neq 0\right)$. Note that for every $\alpha \in G$ one has $|\alpha| \leq h(\alpha)^{g}$. By combining this with (32), (36) and (37) one gets

$$
\begin{aligned}
|x| & \leq\left|x^{\prime}+\beta_{i} y^{\prime}\right| \cdot|z|+\left|\beta_{i}\right| \cdot|y| \\
& \leq\left(|D(F)|^{1 / n(n-1)}+|F(0,1)|^{1 / n}\right) c_{42} .
\end{aligned}
$$

This proves (6) of Theorem 2 . If $(x, y)$ is a solution of $(1)$, then, trivially, $|z| \leq|m|^{1 / n}$. By using this estimate instead of (37) and using again (32) and (36), one obtains (8) of Theorem 3. (We must replace $|m|^{1 / n}+|F(0,1)|^{1 / n}$ by $|m|^{1 / n}\left(1+|F(0,1)|^{1 / n}\right)$ since we must take also solutions $x, y$ with $(x, y)>$ 1 into consideration). This completes the proofs of Theorems 2 and 3.

Proof of the IneQuality (9). Let $P_{1}(X, Y), \ldots, P_{r}(X, Y)$ be the irreducible factors of $F(X, Y)$ in $\mathbb{Z}[X, Y]$ which are not proportional to $Y$. 
Let $\alpha_{i}$ be a root of $P_{i}(X, 1)=0$ in $G$, let $M_{i}=\mathbb{Q}\left(\alpha_{i}\right)$, and let $D_{M_{i}}$ be the discriminant of $M_{i} / \mathbb{Q}$ for $i=1, \ldots, r$. It is proved in [6] (see the proof of $[6$, Theorems 2 and 3]) that

$$
\prod_{i=1}^{r}\left|D_{M_{i}}\right| \leq(n P)^{s n} \cdot|D(F)|_{s}
$$

Further, $G$ is the composite of the conjugates of the fields $M_{1}, \ldots, M_{r}$ over Q. Hence we have, by [23, Lemma 7], that

$$
\left|D_{G}\right| \leq \prod_{i=1}^{r}\left|D_{M_{i}}\right|^{g} .
$$

Now (38), (39) and $g \leq n$ ! imply (9).

\section{Proof of Theorem 4}

In the proof of Theorem 4 we need some notation and two lemmas, one on linear forms in logarithms and one on monic polynomials of given discriminant.

For an algebraic number $\alpha$, we denote by $\mid \alpha$ the maximum of the absolute values of the conjugates of $\alpha$. If $\alpha$ is an algebraic integer of degree $n$, then we have (see, for example, [7]) that

$$
|\alpha| \leq(h(\alpha))^{n} \leq|\alpha|^{n} .
$$

Further, if $\alpha$ and $\beta$ are nonzero algebraic integers in an algebraic number field of degree $n$, then (see [13])

$$
H(\alpha / \beta) \leq(|\alpha|+|\beta|)^{n} .
$$

Let $K$ be an algebraic number field of degree $n$, let $\alpha_{1}, \ldots, \alpha_{k}$ be nonzero elements of $K$, let $A_{1}, \ldots, A_{k}$ be positive numbers with $A_{j} \geq$ $\max \left\{H\left(\alpha_{j}\right), e^{k}\right\}$, let $A=\max \left\{A_{1}, \ldots, A_{k}, e^{e}\right\}$, and let $b_{1}, \ldots, b_{k}$ be rational integers with $B=\max _{1 \leq j \leq k}\left|b_{j}\right|$.

LEMMA 2. If $\Lambda=\alpha_{1}^{b_{1}} \cdots \alpha_{k}^{b_{k}}-1 \neq 0$ then

$$
|\Lambda|>\exp \left\{-c_{43}(k) n^{k+3} \log A_{1} \cdots \log A_{k}(\log k B+\log \log A)\right\}
$$

where $c_{43}(k) \leq 2^{8(k+1)+53}(k+1)^{2(k+1)}$.

Proof. This follows easily from Philippon and Waldschmidt [16, Theorem 1.1] by using a standard argument (see, for example, [19, page 66] or $[16$, page 285$])$. 
LEMMA 3. Let $f \in \mathbb{Z}[X]$ be a monic polynomial of degree $n \geq 2$ with discriminant $0<|D(f)| \leq D(D \geq 2)$. Then there exists $a \in \mathbb{Z}$ such that, for $f^{*}(X)=f(X+a)$,

$$
H\left(f^{*}\right) \leq \exp \left\{c_{44}(n) D^{5(n-1)^{2}}\right\}
$$

with $c_{44}(n)=\left(6 n^{3}\right)^{30 n^{3}}$.

Proof. This is an immediate consequence of Theorem 1 of [9].

Proof of Theorem 4. For brevity, we shall write $D$ and $H$ instead of $D(f)$ and $H(f)$, respectively. Let $\alpha_{1}, \ldots, \alpha_{n}$ be the zeros of $f$ in $\mathbb{C}$, let $K=\mathbb{Q}\left(\alpha_{1}\right)$, and let $x, y, z$ be an arbitrary but fixed solution of

$$
f(x)=y^{z} \text { in } x, y, z \in \mathbb{Z} \quad \text { with }|y|>1, z>1 .
$$

The greatest common divisor of the principal integral ideals $\left[x-\alpha_{1}\right]$ and $\left[\left(x-\alpha_{2}\right) \cdots\left(x-\alpha_{n}\right)\right]$ in $K$ divides the ideal $\left[f^{\prime}\left(\alpha_{1}\right)\right]$. Since $N_{K / Q}\left(f^{\prime}\left(\alpha_{1}\right)\right)=$ $\pm D$, it follows from (13) that there are integral ideals $\mathfrak{a}, \mathfrak{b}, \mathfrak{c}$ in $K$ such that

$$
\mathfrak{a}\left[x-\alpha_{1}\right]=\mathfrak{b} \cdot \mathfrak{c}^{z}
$$

and

$$
\max \left\{N_{K / \mathbb{Q}}(\mathfrak{a}), N_{K / \mathbb{Q}}(\mathfrak{b})\right\} \leq|D| .
$$

Denote by $h, R$ and $D_{K}$ the class number, regulator and discriminant of $K$ over $\mathbb{Q}$. Then, as is known (see, for example, [22, Chapter A]), $D_{K} \mid D$. Further, $\mathfrak{a}^{h}, \mathfrak{b}^{h}, \mathfrak{c}^{h}$ are principal ideals. Then, by a well-known lemma (see, for example, [11, Lemma 3]) and by (17), $\mathfrak{a}^{h}$ and $\mathfrak{b}^{h}$ have generators $\alpha, \beta$ such that

$$
\begin{aligned}
\max \{|\alpha|,|\beta|\} & <\exp \left\{c_{45} h R+\frac{h}{n} \log |D|\right\} \\
& \leq \exp \left\{c_{46}|D|^{1 / 2}(\log |D|)^{n}\right\}
\end{aligned}
$$

with $c_{45}=r\left(6 r n^{2}\right)^{r}, c_{46}=r\left(17 r n^{2}\right)^{n-1}$, where $r$ denotes the unit rank of $K$. Relation (40) yields

$$
\alpha\left(x-\alpha_{1}\right)^{h}=\varepsilon \beta \gamma^{z}
$$


where $\gamma$ is a generator of $c^{h}$ and $\varepsilon$ is a unit in $K$. There exists an independent system $\left\{\eta_{1}, \ldots, \eta_{r}\right\}$ of units for $K$ such that

$$
\prod_{i=1}^{r} \max \left(\log \left|\eta_{i}\right|, 1\right)<c_{47} R,
$$

where $c_{47}=\left(6 r n^{2}\right)^{r}[11$, Lemma 2]. Further, $\varepsilon$ can be written in the form $\rho \eta_{1}^{k_{1}} \ldots \eta_{r}^{k_{r}}$ where $k_{1}, \ldots, k_{r}$ are rational integers and $\rho$ is a unit in $K$ with

$$
|\rho| \leq \exp \left\{c_{46}|D|^{1 / 2}(\log |D|)^{n}\right\}
$$

(see [11, Lemma 3]). Moreover, we can assume by incorporating every $z$ th power in $\gamma$, that $\max _{1 \leq i \leq r}\left|k_{i}\right|<z$ and, for simplicity, we can write $\beta$ in (42) instead of $\rho \beta$, by taking $c_{46}=2 r\left(17 r n^{2}\right)^{n-1}$.

If $|x| \leq H+2$ then (13) implies that

$$
2^{z} \leq|y|^{z} \leq(2 H+3)^{n}
$$

and Theorem 1 is proved. In the sequel we assume that $|x|>H+2$. Then, by $\left|\alpha_{i}\right| \leq H+1$, we have

$$
\left|x-\alpha_{i}\right| \geq 1 \text { for } i=1, \ldots, n
$$

which, together with (13), implies

$$
\left|x-\alpha_{i}\right| \leq|y|^{z} \text { for } i=1, \ldots, n \text {. }
$$

Denote by $\varphi_{i}$ the $\mathbb{Q}$-isomorphisms between the fields $K=\mathbb{Q}\left(\alpha_{1}\right)$ and $\mathbb{Q}\left(\alpha_{i}\right)$, $i=2, \ldots, n$. Then (42) implies

$$
\left(x-\alpha_{i}\right)^{h}=\left(\varphi_{i}\left(\eta_{1}\right)\right)^{k_{1}} \cdots\left(\varphi_{i}\left(\eta_{r}\right)\right)^{k_{r}} \varphi_{i}(\beta / \alpha)\left(\varphi_{i}(\gamma)\right)^{z}
$$

for each $\varphi_{i}$, whence, by (44),

$$
|y|^{z h} \geq \max _{1 \leq i \leq n}\left|x-\alpha_{i}\right|^{h} \geq\left.\bar{\eta}_{1}\right|^{-n z} \cdots\left|\eta_{r}\right|^{-n z}(|\alpha||\beta|)^{-n} \cdot 4^{-(n+1)}|\gamma|^{z} .
$$

Hence

$$
\left.\left.|\eta| y\right|^{h}\left|\eta_{1}\right|^{n} \cdots \eta_{r}\right|^{n} 4^{(n+1) / z}(|\alpha| \beta \mid)^{n / z} .
$$

Together with (41), (43) and (17), this gives

$$
\log H\left(\gamma / \varphi_{2}(\gamma)\right)<n \log 2+\log |\gamma|<2 n c_{46}|D|^{1 / 2}(\log |D|)^{n} \log |y| .
$$

We may assume without loss of generality that

$$
\min _{\substack{i, j \\ i \neq j}} \frac{\left|\alpha_{i}-\alpha_{j}\right|}{\left|x-\alpha_{i}\right|}=\frac{\left|\alpha_{2}-\alpha_{1}\right|}{\left|x-\alpha_{2}\right|} .
$$


It follows from (13) and

$$
|D|=\prod_{\substack{1 \leq i, j \leq n \\ i \neq j}}\left|\alpha_{i}-\alpha_{j}\right|
$$

that

$$
\prod_{\substack{1 \leq i, j \leq n \\ i \neq j}} \frac{\left|\alpha_{i}-\alpha_{j}\right|}{\left|x-\alpha_{i}\right|}=\frac{|D|}{|y|^{2(n-1)}}
$$

whence, by (46),

$$
\left|\frac{\alpha_{2}-\alpha_{1}}{x-\alpha_{2}}\right| \leq \frac{|D|}{|y|^{2 / n}}
$$

Now (46) implies that $\left|x-\alpha_{1}\right| \leq\left|x-\alpha_{2}\right|$. Further, we may assume that $|y|^{z}>(h|D|)^{2 n}$, for otherwise our theorem is proved in view of (17). Hence, by (47), we have

$$
\left|\left(\frac{x-\alpha_{1}}{x-\alpha_{2}}\right)^{h}-1\right| \leq\left|\frac{x-\alpha_{1}}{x-\alpha_{1}}-1\right| \cdot h<\frac{|D| h}{|y|^{z / n}}<\frac{1}{|y|^{z / 2 n}} .
$$

In the next step we show that $\left(\left(x-\alpha_{1}\right) /\left(x-\alpha_{2}\right)\right)^{h}=1$ implies $z \leq$ $n^{2} \log _{2}|D|$. Indeed, in this case $\left(\alpha_{1}-\alpha_{2}\right) /\left(x-\alpha_{1}\right)$ must be an algebraic integer, and hence, by (13),

$$
2^{z} \leq|y|^{z}=\left|N_{K / \mathbb{Q}}\left(x-\alpha_{1}\right)\right| \leq\left|N_{L / Q}\left(\alpha_{1}-\alpha_{2}\right)\right| \leq|D|^{n^{2}}
$$

where $L=\mathbb{Q}\left(\alpha_{1}, \alpha_{2}\right)$.

If $\left|\left(\left(x-\alpha_{1}\right) /\left(x-\alpha_{2}\right)\right)^{h}-1\right|$ is nonzero then we use Lemma 2 together with (41), (43), (45) and (17) to deduce from the relation

$$
\begin{aligned}
0 & <\left|\left(\frac{x-\alpha_{1}}{x-\alpha_{2}}\right)^{h}-1\right| \\
& =\left|\left(\frac{\eta_{1}}{\varphi_{2}\left(\eta_{1}\right)}\right)^{k_{1} h} \cdots\left(\frac{\eta_{r}}{\varphi_{2}\left(\eta_{r}\right)}\right)^{k_{r} h}\left(\frac{\beta / \alpha}{\varphi_{2}(\beta / \alpha)}\right)\left(\frac{\gamma}{\varphi_{2}(\gamma)}\right)^{z h}-1\right|
\end{aligned}
$$

that

$$
\left|\left(\frac{x-\alpha_{1}}{x-\alpha_{2}}\right)^{h}-1\right|>\exp \left\{-c_{48}|D|^{3 / 2}(\log |D|)^{3 n+1} \log |y| \log z\right\}
$$


where $c_{48} \leq 2^{20 n+49}(n+2)^{12 n-2}$. Now comparing (48) and (49), we see that

$$
z<2^{20(n+3)}(n+2)^{15 n}|D|^{2}
$$

follows.

\section{References}

[1] A. Baker, 'Contributions to the theory of diophantine equations', Philos. Trans. Roy. Soc. London A 263 (1968), 173-208.

[2] A. Baker, 'Bounds for the solutions of the hyperelliptic equation', Proc. Cambridge Philos. Soc. 65 (1969), 439-444.

[3] B. Brindza, 'Thue equations and multiplicative independence', Number theory and cryptography (J. H. Loxton, ed.), Cambridge Univ. Press, 1990, pp. 213-220.

[4] J. Coates, 'An effective $p$-adic analogue of a theorem of Thue', Acta Arith. 15 (1969), 279-305.

[5] J. H. Evertse and K. Györy, 'Decomposable form equations', New Advances in Transcendence Theory (A. Baker, ed.), Cambridge Univ. Press, 1988, pp. 175-202.

[6] J. H. Evertse and K. Gyory, 'Effective finiteness results for binary forms with given discriminant', to appear.

[7] J. H. Evertse, K. Györy, C. L. Stewart and R. Tijdeman, 'On $S$-unit equations', Invent. Math. 92 (1988), 461-477.

[8] K. Györy, 'Sur les polynômes à coefficients entiers et de discriminant donné II.', Publ. Math. Debrecen 21 (1974), 125-144.

[9] K. Györy, 'On polynomials with integer coefficients and given discriminant IV.', Publ. Math. Debrecen 25 (1978), 155-167.

[10] K. Györy, 'On the number of solutions of linear equations in units of an algebraic number field', Comment. Math. Helv. 54 (1979), 583-600.

[11] K. Györy, 'On the solutions of linear diophantine equations in algebraic integers of bounded norm', Ann. Univ. Sci. Budapest Eötvös Sect. Math. 22/23 (1980), 225-233.

[12] K. Györy, 'Explicit upper bounds for the solutions of some diophantine equations', Ann. Acad. Sci. Fenn. Ser. A I 5 (1980), 3-12.

[13] K. Györy, 'Résultats effectifs sur la représentation des entiers par des formes décomposables', Queen's Papers in Pure and Applied Math., No. 56, Kingston, Canada, 1980.

[14] K. Györy, 'On $S$-integral solutions of norm form, discriminant form and index form equations', Studia Sci. Math. Hungar. 16 (1981), 149-161.

[15] S. Lang, 'Fundamentals of diophantine geometry', Springer-Verlag, 1983.

[16] P. Philippon and M. Waldschmidt, 'Lower bounds for linear forms in logarithms', New Advances in Transcendence Theory (A. Baker, ed.), Cambridge Univ. Press, 1988, pp. 280-312.

[17] J. B. Rosser and L. Schoenfeld, 'Approximate formulas for some functions of prime numbers', Illinois J. Math. 6 (1962), 64-94.

[18] A. Schinzel and R. Tijdeman, 'On the equation $y^{m}=P(x)$ ', Acta Arith. 31 (1976), 199-204.

[19] T. N. Shorey, A. J. van der Poorten, R. Tijdeman and A. Schinzel, 'Applications of the Gel'fond-Baker method to diophantine equations', Transcendence Theory: Advances and Applications (A. Baker and D. W. Masser, eds.), Academic Press, London, 1977, pp. 59-77.

[20] T. N. Shorey and R. Tijdeman, Exponential diophantine equations, Cambridge Univ. Press, 1986. 
[21] C. L. Siegel, 'Abschätzung von Einheiten', Nachr. Akad. Wiss. Göttingen, Math.-Phys. Kl. 2 (1969), 71-86.

[22] V. G. Sprindžuk, Classical diophantine equations in two unknowns, Nauka, Moskva, 1982. (Russian)

[23] H. M. Stark, 'Some effective cases of the Brauer-Siegel theorem', Invent. Math. 23 (1974), 135-152.

[24] L. A. Trelina, 'Representations of powers by polynomials in algebraic number fields', Dokl. Akad. Nauk BSSR 29 (1985), 5-8, in Russian.

[25] R. Zimmert, 'Ideale kleiner Norm in Idealklassen und eine Regulatorabschätzung', Invent. Math. 62 (1981), 367-380.

Mathematical Institute

Kossuth Lajos University

4010 Debrecen

Hungary
Department of Mathematics and

Computer Science 2300 RA Leiden

P.O. Box 9512

The Netherlands 